\title{
PIECEWISE LINEAR FUNCTIONS WITH ALMOST ALL POINTS EVENTUALLY PERIODIC
}

\author{
MELVYN B. NATHANSON
}

\begin{abstract}
Let $f:[0,1] \rightarrow[0,1]$ be continuous, and let $f^{p}$ denote the $p$ th iterate of $f$. Li and Yorke [2] proved that if there is a point $x \in[0,1]$ such that $f^{3}(x)=x$ but $f(x) \neq x$, then $f$ is chaotic in the sense that $f$ has periodic points of arbitrarily large period, and uncountably many points which are not even asymptotically periodic. But this chaos can be measure theoretically trivial. For each $p \geqslant 3$ we construct a continuous, piecewise linear function $f:[0,1] \rightarrow[0,1]$ such that $f$ is chaotic, but almost every point of $[0,1]$ has eventual period $p$. The condition "eventual period $p$ " cannot be replaced by "period $p$ ". We prove that if $f^{p}(x)=x$ for almost all $x \in[0,1]$, then $f^{2}(x)=x$ for all $x \in[0,1]$. Moreover, we describe a normal form for all such "square roots of the identity."
\end{abstract}

Let $f:[0,1] \rightarrow[0,1]$ be continuous. The iterates of $f$ are defined as follows: $f^{0}(x)=x$ and $f^{n}(x)=f\left(f^{n-1}(x)\right)$ for $n=1,2,3, \ldots$ The point $x \in[0,1]$ is periodic under $f$ with period $p$ if $f^{p}(x)=x$ but $f^{k}(x) \neq x$ for $k=1,2$, $\ldots, p-1$. If $f^{n}(x)$ has period $p$ for some $n$, then $x$ is eventually periodic under $f$ with period $p$. Li and Yorke [2] have recently obtained the remarkable result that if $f:[0,1] \rightarrow[0,1]$ has a point of period three, then $f$ is "chaotic" in the sense that, first, there are points $x \in[0,1]$ of arbitrarily large period (in fact, of all periods), and, second, there is an uncountable set $S \subset[0,1]$ such that no point of $S$ is even asymptotically periodic (that is, if $y \in S$ and if $x$ $\in[0,1]$ is periodic, then $\left.\lim \sup \left|f^{n}(y)-f^{n}(x)\right|>0\right)$, and such that, if $y_{1}, y_{2}$ are any two points of $S$, then $\lim \inf \left|f^{n}\left(y_{1}\right)-f^{n}\left(y_{2}\right)\right|=0$ and $\lim \sup \left|f^{n}\left(y_{1}\right)-f^{n}\left(y_{2}\right)\right|>0$. More generally, Li and Yorke proved that if there is a point $x \in[0,1]$ such that either $f^{3}(x) \leqslant x<f(x)<f^{2}(x)$ or $f^{3}(x)$ $\geqslant x>f(x)>f^{2}(x)$, then $f$ is chaotic. By a combinatorial argument, Nathanson [7] extended this result to show that if $f$ has a point of period five or seven, then $f$ is chaotic. Ulam, May, Oster, and others [1], [3]-[6], [8] have studied in detail the iterations of nonlinear functions $f$ and the dependence of the trajectories $x, f(x), f^{2}(x), f^{3}(x), \ldots$ on the initial value $x$.

The object of this note is to show that, from the point of view of Lebesgue measure, the results on chaos can be misleading. For every $p \geqslant 3$ we shall construct a continuous, piecewise linear function $f:[0,1] \rightarrow[0,1]$ such that almost every $x \in[0,1]$ has eventual period $p$. Moreover, $f$ will be chaotic. This

Received by the editors January 19, 1976.

AMS (MOS) subject classifications (1970). Primary 26A18; Secondary 39A20.

Key words and phrases. Iterations, nonlinear functions, chaotic functions, dynamical systems. 
result is best possible in the sense that the word "eventual" cannot be removed from the statement of the theorem. For if almost every point $x \in[0,1]$ has period $p$ under $f$, then the continuity of $f$ implies that $f^{p}(x)=x$ for all $x$, and so $f^{p}=$ identity. However, we shall prove that if $f^{p}=$ identity, then $f^{2}=$ identity. Moreover, we shall describe a normal form for all such square roots of the identity.

Theorem 1. Let $p \geqslant 3$ and let $\delta \in\left(0,2^{-p}\right)$. Define $f:[0,1] \rightarrow[0,1]$ in the following way:

$$
f(x)= \begin{cases}x+1 / p, & 0 \leqslant x \leqslant(p-1) / p \\ 1-(1-\delta) \delta^{-1}(x-(p-1) / p), & (p-1) / p<x<(p-1) / p+\delta \\ x-(p-1) / p, & (p-1) / p+\delta \leqslant x \leqslant 1\end{cases}
$$

Then $f$ is continuous, piecewise linear, chaotic, and almost every point $x \in[0,1]$ has eventual period $p$ under $f$.

Proof. Clearly, $f$ is continuous and piecewise linear. By the theorem of $\mathrm{Li}$ and Yorke, $f$ is also chaotic, since

$$
f^{3}\left(\frac{p-2}{p}\right)=\frac{1}{p} \leqslant \frac{p-2}{p}<f\left(\frac{p-2}{p}\right)=\frac{p-1}{p}<f^{2}\left(\frac{p-2}{p}\right)=1 .
$$

Let $C=\cup_{i=1}^{p}[(i-1) / p+\delta, i / p]$. For $i=1,2, \ldots, p-1$, the function $f$ maps the interval $[(i-1) / p+\delta, i / p]$ linearly onto $[i / p+\delta,(i+1) / p]$ by the rule $f(x)=x+1 / p$. Also, $f$ maps the interval $[(p-1) / p+\delta, 1]$ linearly onto the interval $[\delta, 1 / p]$ by the rule $f(x)=x-(p-1) / p$. Thus, each point $x \in C$ has period $p$, and $f(C)=C$. Let $x \in[0,1]$. If $f^{m}(x) \in C$ for some $m$, then $f^{n}(x) \in C$ for all $n \geqslant m$, and $x$ has tventual period $p$. We define

$$
\begin{aligned}
& C^{*}=\left\{x \in[0,1] \mid f^{m}(x) \in C \text {, for some } m\right\}, \\
& U^{*}=\left\{x \in[0,1] \mid f^{n}(x) \notin C \text { for } n=0,1,2,3, \ldots\right\} .
\end{aligned}
$$

The sets $C^{*}$ and $U^{*}$ partition $[0,1]$. Every point of $C^{*}$ has eventual period $p$. If $x \in[0,1]$ does not have eventual period $p$, then $u \in U^{*}$. Clearly, $U^{*}$ $\subset \cup_{i=0}^{p-1}(i / p, i / p+\delta) \cup\{0\}$. Let $\mu(X)$ denote the Lebesgue measure of $X$. We shall prove that $\mu\left(C^{*}\right)=1$, or, equivalently, that $\mu\left(U^{*}\right)=0$.

We begin by studying the open interval $U_{0}=((p-1) / p,(p-1) / p+\delta)$. Let $U_{n}=\left\{x \in U_{0} \mid f^{n}(x) \notin C\right\}$. Clearly, $U_{0} \supset U_{1} \supset U_{2} \supset U_{3} \supset \cdots$.

Let $\lambda=\delta /(1-\delta)$. We shall prove, by induction on $n$, that each $U_{n}$ is a union of disjoint open intervals whose lengths are of the form $\delta \lambda^{k}$ for $k=0$, $1,2, \ldots, n$, and that $f^{n}$ maps each of these intervals linearly onto one of the $p-1$ intervals $(i / p, i / p+\delta)$ for $i=1,2, \ldots, p-1$. Moreover, if $A_{k, n}^{(i)}$ denotes the number of open intervals of length $\delta \lambda^{i}$ of $U_{n}$ which $f^{n}$ maps onto $(i / p, i / p+\delta)$, then the integers $A_{k, n}^{(i)}$ can be computed by the following rules: 


$$
\begin{aligned}
& A_{0,0}^{(i)}= \begin{cases}1 & \text { if } i=p-1, \\
0 & \text { if } i=1,2, \ldots, p-2 ;\end{cases} \\
& A_{k, n}^{(i)}=\sum_{j=1}^{i} A_{k-1, n-j}^{(p-1)} \text { for } n=1,2, \ldots,
\end{aligned}
$$

where $A_{k, n}^{(i)}=0$ if $k<0$ or $n<0$.

These statements are obviously true for $n=0$. Let us assume that they hold for some $n-1 \geqslant 0$. We want to describe the structure of $U_{n}$. Since $U_{n}$ $\subset U_{n-1}$, it is enough to understand how $f^{n}$ acts on the intervals that make up $U_{n-1}$. Let $I$ be an open interval of $U_{n-1}$ of length $\delta \lambda^{k}$, where $k \leqslant n-1$. If $f^{n-1}$ maps $I$ linearly onto $(i / p, i / p+\delta)$ for some $i=1,2, \ldots, p-2$, then $f^{n}$ maps $I$ linearly onto $((i+1) / p,(i+1) / p+\delta)$. If $f^{n-1}$ maps $I$ linearly onto $((p-1) / p,(p-1) / p+\delta)$, then $f^{n}$ maps $I$ linearly onto $(\delta, 1)$. Since the length of $I$ is $\delta \lambda^{k}$, it follows that the slope of $f^{n}$ on $I$ has absolute value $(1-\delta) / \delta \lambda^{k}=1 / \lambda^{k+1}$. Moreover, for each $i=1,2, \ldots, p-1$ there is exactly one open interval of length $\delta \lambda^{k+1}$ of $I$ which $f^{n}$ maps linearly onto $(i / p, i / p+\delta)$. The function $f^{n}$ sends the complement of these $p-1$ intervals into $C$. It follows that $A_{k, n}^{(1)}=A_{k-1, n-1}^{(p-1)}$ and $A_{k, n}^{(i)}=A_{k, n-1}^{(i-1)}+A_{k-1, n-1}^{(p-1)}$ for $i$ $=2,3, \ldots, p-1$. These relations imply that $A_{k, n}^{(i)}=\sum_{j=1}^{i} A_{k-1, n-j}^{(p-1)}$. This completes the induction.

Now we can compute the measure of the sets $U_{n}$. It follows from the definition of the numbers $A_{k, n}^{(i)}$ that

$$
\mu\left(U_{n}\right)=\sum_{i=1}^{p-1} \sum_{k=0}^{n} A_{k, n}^{(i)} \delta \lambda^{k}=\delta \sum_{i=1}^{p-1} P_{n}^{(i)}(\lambda)
$$

where $P_{n}^{(i)}(x)$ is the polynomial defined by

$$
P_{n}^{(i)}(x)=\sum_{k=0}^{n} A_{k, n}^{(i)} x^{k}
$$

The recurrence relations for the coefficients $A_{k, n}^{(i)}$ imply that

$$
\begin{aligned}
& P_{0}^{(i)}(x)= \begin{cases}1 & \text { if } i=p-1, \\
0 & \text { if } i=1,2, \ldots, p-2 ;\end{cases} \\
& P_{n}^{(i)}(x)=x \sum_{j=1}^{i} P_{n-j}^{(p-1)}(x) \text { for } n=1,2, \ldots,
\end{aligned}
$$

where $P_{n}^{(i)}(x)=0$ for $n<0$.

Clearly, the degree of $P_{n}^{(i)}(x)$ is $n$. Write $n$ in the form $n=q(p-1)-r$, where $r=0,1,2, \ldots, p-2$. I claim that $P_{n}^{(p-1)}(x)$ is divisible by $x^{q}$. This is certainly true for $q=0$ and $q=1$, since $P_{n}^{(p-1)}(x)$ is divisible by $x$ for $n=1,2, \ldots, p-1$. Moreover, the recurrence relation implies that if $x^{k}$ divides $P_{m}^{(p-1)}(x)$, then $x^{k}$ divides $P_{n}^{(p-1)}(x)$ for all $n \geqslant m$. Let us assume the claim is true for some $q-1 \geqslant 1$ and $r=0,1, \ldots, p-2$. If $n=q(p-1)$ $-r$, then $n-(p-1)=(q-1)(p-1)-r$, and so $P_{n-(p-1)}^{(p-1)}(x)$ is divisible by 
$x^{q-1}$. Consequently, $P_{n-j}^{(p-1)}(x)$ is divisible by $x^{q-1}$ for $j=1,2, \ldots, p-1$. Since $P_{n}^{(p-1)}(x)=x \sum_{j=1}^{p-1} P_{n-j}^{(p-1)}(x)$, it follows that $P_{n}^{(p-1)}(x)$ is divisible by $x \cdot x^{q-1}=x^{q}$. The claim follows by induction on $q$.

Since the coefficients of $P_{n}^{(p-1)}(x)$ are nonnegative, it follows that for $0<\lambda<1$ we have

$$
P_{n}^{(i)}(\lambda) \leqslant P_{n}^{(p-1)}(\lambda) \leqslant \lambda^{(n+r) /(p-1)} P_{n}^{(p-1)}(1) \leqslant \lambda^{n /(p-1)} P_{n}^{(p-1)}(1)
$$

where $i=1,2,3, \ldots, p-1$ and $n=q(p-1)-r$. But the integers $P_{n}^{(p-1)}(1)$ satisfy the recurrence relations

$$
P_{0}^{(p-1)}(1)=1, \quad P_{n}^{(p-1)}(1)=\sum_{j=1}^{p-1} P_{n-j}^{(p-1)}(1) \quad \text { for } n=1,2, \ldots,
$$

where $P_{n}^{(p-1)}(1)=0$ for $n<0$. An easy induction shows that $P_{n}^{(p-1)}(1)$ $\leqslant 2^{n}$ for $n=0,1,2, \ldots$ Therefore,

$$
P_{n}^{(i)}(\lambda) \leqslant\left(2 \lambda^{1 /(p-1)}\right)^{n}
$$

But for $0<\delta<2^{-p}$ we have

$$
0<\lambda^{1 /(p-1)}=\left(\frac{\delta}{1-\delta}\right)^{1 /(p-1)}<(2 \delta)^{1 /(p-1)}<\frac{1}{2}
$$

and so $0<2 \lambda^{1 /(p-1)}<1$. Therefore,

$$
\mu\left(U_{n}\right)=\delta \sum_{i=1}^{p-1} P_{n}^{(i)}(\lambda) \leqslant \delta(p-1)\left(2 \lambda^{1 /(p-1)}\right)^{n}
$$

Consequently,

$$
\lim _{n \rightarrow \infty} \mu\left(U_{n}\right)=0 .
$$

Let us return to the set $U^{*}=\left\{x \in[0,1] \mid f^{n}(x) \notin C\right.$ for $\left.n=0,1,2, \ldots\right\}$. Let $U_{n}^{*}=\left\{x \in[0,1] \mid f^{n}(x) \notin C\right\}$. Then

$$
U_{0}^{*}=\bigcup_{i=0}^{p-1}\left(\frac{i}{p}, \frac{i}{p}+\delta\right) \cup\{0\} \supset U_{1}^{*} \supset U_{2}^{*} \supset \cdots
$$

and $U^{*}=\cap_{n=0}^{\infty} U_{n}^{*}$. Therefore, $\mu\left(U^{*}\right)=\lim _{n \rightarrow \infty} \mu\left(U_{n}^{*}\right)$, Since $f(0)=1 / p$ $\in C$, we have $0 \notin U_{n}^{*}$ for $n \geqslant 1$. For $i=0,1, \ldots, p-1$, let $U_{n}^{(i)}=\{x$ $\left.\in(i / p, i / p+\delta) \mid f^{n}(x) \notin C\right\}$. Then $U_{n}^{(p-1)}=U_{n}$ and $U_{n}^{*}=\cup_{i=0}^{p-1} U_{n}^{(i)}$.

For $i=0,1,2, \ldots, p-2$, the map $f^{p-1-i}$ sends $U_{0}^{(i)}=(i / p, i / p+\delta)$ linearly onto $((p-1) / p,(p-1) / p+\delta)=U_{0}^{(p-1)}=U_{0}$ according to the rule $f^{p-1-i}(x)=x+(p-1-i) / p$. Therefore,

$$
U_{n}^{(i)}=\left\{x-(p-1-i) / p \mid x \in U_{n-p+1+i}\right\}
$$


for $n \geqslant p-1-i$, and so $\mu\left(U_{n}^{(i)}\right)=\mu\left(U_{n-p+1+i}\right)$. Since $U_{n}^{*}=\cup_{i=0}^{p-1} U_{n}^{(i)}$, we have

and so

$$
\mu\left(U_{n}^{*}\right)=\sum_{i=0}^{p-1} \mu\left(U_{n}^{(i)}\right)=\sum_{i=0}^{p-1} \mu\left(U_{n-p+1+i}\right)
$$

$$
\mu\left(U^{*}\right)=\lim _{n \rightarrow \infty} \mu\left(U_{n}^{*}\right)=0 .
$$

This completes the proof of the theorem.

THEOREM 2. If $f:[0,1] \rightarrow[0,1]$ is a continuous function such that $f^{p}(x)=x$ for all $x$, then $f^{2}(x)=x$ for all $x$. In particular, if $p$ is odd, then $f(x)=x$ for all $x$.

Proof. If $f^{p}(x)=x$ for all $x$, then $f$ is a continuous bijection of $[0,1]$, and so $f$ is monotone and either $f(0)=0, f(1)=1$ or $f(0)=1, f(1)=0$.

Let $f$ be a monotone function such that $f(0)=0, f(1)=1$. If $f(x) \neq x$ for some $x \in(0,1)$, say, $f(x)>x$, then there is an interval $[a, b]$ with $0 \leqslant a<x$ $<b \leqslant 1$ such that $a<x<f(x)<b$ for all $x \in(a, b)$. Then

$$
0 \leqslant a<x<f(x)<f^{2}(x)<\cdots<f^{p-1}(x)<f^{p}(x)<\cdots<b \leqslant 1
$$

and so $f^{p}(x) \neq x$. Therefore, if $f^{p}(x)=x$ for all $x$, and $f(0)=0, f(1)=1$, then $f(x)=x$ for all $x$.

Let $f$ be a monotone function such that $f(0)=1, f(1)=0$. If $p$ is odd, then $f^{p}(0)=1$. Therefore, if $f^{p}(x)=x$ for all $x$, then $p=2 q$ is even. Let $g(x)=f^{2}(x)$. Then $g$ is a monotone function such that $g(0)=0, g(1)=1$, and $g^{q}(x)=f^{p}(x)=x$. Therefore, $g(x)=f^{2}(x)=x$ for all $x \in[0,1]$. This proves the theorem.

The next result shows that all square roots of the identity are obtained by conjugating the function $h(x)=1-x$ by an increasing, "half-linear" function $\gamma$. This observation is due to David Kazhdan.

THEOREM 3. Let $f:[0,1] \rightarrow[0,1]$ be a continuous function such that $f(0)=1$, $f(1)=0$, and $f^{2}(x)=x$ for all $x \in[0,1]$. Then there is a unique increasing function $\gamma:[0,1] \rightarrow[0,1]$ with $\gamma$ linear on $\left[0, \frac{1}{2}\right]$ such that

$$
f(x)=\gamma\left(1-\gamma^{-1}(x)\right)
$$

for all $x \in[0,1]$.

Proof. Clearly, $f$ is a monotone decreasing function on $[0,1]$. Let $a \in(0,1)$ be a fixed point of $f$. If $x<a$, then $f(x)>f(a)=a>x$. If $x>a$, then $f(x)$ $<f(a)=a<x$. Therefore, $a$ is the unique fixed point of $f$.

We define the function $\gamma$ on $[0,1]$ in the following way:

$$
\gamma(x)= \begin{cases}2 a x, & 0 \leqslant x \leqslant \frac{1}{2}, \\ f(2 a(1-x)), & \frac{1}{2} \leqslant x \leqslant 1 .\end{cases}
$$


Observe that $f(2 a(1-x))$ increases monotonically from $a$ to 1 as $x$ increases from $\frac{1}{2}$ to 1 . Therefore, $\gamma$ has a continuous inverse on $[0,1]$, and $\gamma^{-1}(x)$ $=x / 2 a$ for $x \in[0, a]$.

Suppose $0 \leqslant x \leqslant a$. Then $1-\gamma^{-1}(x)=1-x / 2 a \in\left[\frac{1}{2}, 1\right]$, and so

$$
\gamma\left(1-\gamma^{-1}(x)\right)=\gamma(1-x / 2 a)=f(2 a(1-(1-x / 2 a)))=f(x) .
$$

Suppose $a \leqslant x \leqslant 1$. Then

$$
\gamma^{-1}(x)=y \in\left[\frac{1}{2}, 1\right] \text { and } x=\gamma(y)=f(2 a(1-y)) .
$$

Therefore, $f(x)=f^{2}(2 a(1-y))=2 a(1-y)$. On the other hand, $1-y$ $\in\left[0, \frac{1}{2}\right]$ and so

$$
\gamma\left(1-\gamma^{-1}(x)\right)=\gamma(1-y)=2 a(1-y)=f(x) .
$$

This proves that $f(x)=\gamma\left(1-\gamma^{-1}(x)\right)$ for all $x \in[0,1]$.

Let $\delta:[0,1] \rightarrow[0,1]$ be linear on $\left[0, \frac{1}{2}\right]$ and satisfy $f(0)=0$ and

$$
f(x)=\delta\left(1-\delta^{-1}(x)\right)
$$

for all $x \in[0,1]$. Let $\delta\left(\frac{1}{2}\right)=b$. Then

$$
f(b)=\delta\left(1-\delta^{-1}(b)\right)=\delta\left(\frac{1}{2}\right)=b
$$

and so $b$ is a fixed point of $f$. But $f$ has the unique fixed point $a$. Therefore, $a=b$ and $\delta(x)=2 a x=\gamma(x)$ for $x \in\left[0, \frac{1}{2}\right]$.

If we replace $x$ by $\gamma(x)$ in (1) and (2), we obtain

$$
\gamma(1-x)=\delta\left(1-\delta^{-1} \gamma(x)\right)
$$

for all $x \in[0,1]$. Suppose $\frac{1}{2} \leqslant x \leqslant 1$. Then $\gamma(x) \in[a, 1]$ and $\delta^{-1} \gamma(x) \in\left[\frac{1}{2}\right.$, 1]. Therefore,

$$
2 a(1-x)=\gamma(1-x)=\delta\left(1-\delta^{-1} \gamma(x)\right)=2 a\left(1-\delta^{-1} \gamma(x)\right)
$$

and so $x=\delta^{-1} \gamma(x)$ and $\gamma(x)=\delta(x)$ for $x \in\left[\frac{1}{2}, 1\right]$. This proves the theorem.

\section{REFERENCES}

1. J. Guckenheimer, G. F. Oster and A. Ipaktchi, The dynamics of density dependent population models (preprint).

2. T.-Y. Li and J. A. Yorke, Period three implies chaos, Amer. Math. Monthly 82 (1975), 985-992.

3. R. M. May, Biological populations with nonoverlapping generations: stable points, stable cycles, and chaos, Science 186 (1974), 645-647.

4. R. M. May and G. F. Oster, Bifurcations and dynamic complexity in simple ecological models, Amer. Natur. 110 (1976), 573-599.

5. N. Metropolis, M. L. Stein and P. R. Stein, Stable states of a non-linear transformation, Numer. Math. 10 (1967), 1-19. MR 37 \# 7068. 
6. - On finite limit sets for transformations on the unit interval, J. Combinatorial Theory Ser. A 15 (1973), 25-44. MR 47 \#5183.

7. M. B. Nathanson, Permutations, periodicity, and chaos, J. Combinatorial Theory Ser. A 21 (1976) (to appear).

8. P. R. Stein and S. M. Ulam, Non-linear transformation studies on electronic computers, Rozprawy Mat. 39 (1964), 1-66. MR 29 \#6666.

Department of Mathematics, Brooklyn College, CUNY, Brooklyn, New York 11210

Current address: Department of Mathematics, Southern Illinois University, Carbondale, Illinois 62901 